\title{
Stability and evolution of the climate system of Mars
}

\author{
Takasumi Nakamura and Eiichi Tajika \\ Department of Earth and Planetary Science, The University of Tokyo, 7-3-1 Hongo, Bunkyo-ku, Tokyo 113-0033, Japan
}

(Received December 4, 2000; Revised April 14, 2001; Accepted April 17, 2001)

\begin{abstract}
We construct a one-dimensional energy balance climate model for Mars which incorporates greenhouse effect of $\mathrm{CO}_{2}$ and latitudinal heat transport so that we can express a latitudinal temperature gradient and change of an areal extent of a polar ice cap. By considering energy balance and $\mathrm{CO}_{2}$ budget among atmosphere, ice caps, and regolith, we investigate stability and evolution of the climate system of Mars. Under the present condition there are two stable steady state solutions of the system. One corresponds to a partial ice-covered solution (the present state), and the other is a warmer ice-free solution. Although this is also predicted by previous studies, these solutions are qualitatively different from them. When we assume $\mathrm{CO}_{2}$ as a dominant greenhouse gas for a warm and wet climate on the early Mars, we found that the total amount of $\mathrm{CO}_{2}$ within the whole system should have been larger than that at present and have decreased by some removal processes. We also found that a climate jump must have occurred during the evolution from the early warm climate to the present state, and ice caps on the early Mars might have extended to the mid-latitude. The atmospheric pressure may have decreased further after the climate jump.
\end{abstract}

\section{Introduction}

Ever since Mariner 9 and Vikings found many valleys and basins on the surface of Mars, Martian paleoenvironment under which these topographies were created has been discussed. Valley networks and traces of possible paleoshorelines on the Martian surface are regarded as possible evidence of existence of liquid water during the early history of Mars (e.g., Goldspiel and Squyres, 1991; Baker et al., 1992; Head et al., 1999). The low elevation of the northern hemisphere and its flatness might also imply that the northern hemisphere was once covered with a vast ocean (Smith et al., 1998). These features suggest that the ancient Mars might have warm and wet environment. Because solar luminosity was lower in the past than at present (Sagan and Mullen, 1972), it is likely that strong greenhouse effect due to some greenhouse gas species should have worked at that time to make warm environment on the ancient Mars. It was generally believed that the Martian atmosphere was reducing early in its history. However, it has been shown that reducing gases (such as $\mathrm{CH}_{4}$ and $\mathrm{NH}_{3}$ ) are photochemically unstable (Kuhn and Atreya, 1979). It is now believed that $\mathrm{CO}_{2}$ was the dominant greenhouse gas in the early atmosphere of Mars, although other possibilities such as greenhouse effect of $\mathrm{CH}_{4}$ and/or $\mathrm{NH}_{3}$ cannot be excluded entirely (Kasting, 1997; Sagan and Chyba, 1997).

There are several controlling mechanisms for the atmospheric $\mathrm{CO}_{2}$ pressure on Mars. For instance, if there was liquid water (oceans or ponds) under warm and wet conditions, $\mathrm{CO}_{2}$ must have removed from the atmosphere by chemical weathering of silicate rocks followed by precipitation of carbonate (Pollack et al., 1987; Baker et al., 1991). Even if the

Copy right (c) The Society of Geomagnetism and Earth, Planetary and Space Sciences (SGEPSS); The Seismological Society of Japan; The Volcanological Society of Japan; The Geodetic Society of Japan; The Japanese Society for Planetary Sciences. surface temperature was below the freezing point of water, large impacts could have removed a part of the atmosphere during the heavy bombardment period (Melosh and Vickery, 1989). Escape of atmospheric $\mathrm{CO}_{2}$ by ion sputtering is also suggested to have occurred throughout the history of Mars (Luhmann et al., 1992). On the other hand, volcanic degassing might have supplied $\mathrm{CO}_{2}$ to the atmosphere (Baker et al., 1991). It is also important to consider the polar ice caps (e.g., Leighton and Murray, 1966) and the surface regolith (Fanale and Cannon, 1974) which might have been large $\mathrm{CO}_{2}$ reservoirs to exchange $\mathrm{CO}_{2}$ with the atmosphere.

Gierasch and Toon (1973) assumed the atmosphere and the polar ice caps as dominant $\mathrm{CO}_{2}$ reservoirs. They studied multiple solutions for a model of the atmosphere-ice cap (AI) system and analyzed stability of the solutions. They used a zero-dimensional energy balance climate model, although they assumed the winter polar region and the low-latitude region and considered heat transport between them due to baroclinic instability. They found that the AI system has two stable solutions: one is the present state and the other is the warmer state with higher $\mathrm{CO}_{2}$ pressure.

On the other hand, McKay et al. (1991) assumed the atmosphere and the regolith as dominant $\mathrm{CO}_{2}$ reservoirs, and studied multiple solutions for the atmosphere-regolith (AR) system and stability of solutions. They combined a onedimensional radiative-convective model proposed by Pollack et al. (1987) with a model for $\mathrm{CO}_{2}$ exchange between the regolith and the atmosphere proposed by Toon et al. (1980). They argued that the AR system may have two stable states, depending on sensitivity of $\mathrm{CO}_{2}$ adsorption by the regolith to the surface temperature. One of them is the present state, and the other is the warmer state with higher $\mathrm{CO}_{2}$ pressure.

These two studies, however, may have some problems. Gierasch and Toon (1973) did not consider the greenhouse 
effect of $\mathrm{CO}_{2}$ and adsorption of $\mathrm{CO}_{2}$ by the regolith, and assumed the polar cap to be an infinite $\mathrm{CO}_{2}$ reservoir with no areal extent. On the other hand, McKay et al. (1991) did not consider roles of $\mathrm{CO}_{2}$-ice caps as a $\mathrm{CO}_{2}$ reservoir which may regulate the atmospheric $\mathrm{CO}_{2}$ pressure and as a reflector of incident solar flux, especially in the distant past.

Haberle et al. (1994) modified the energy balance model of Gierasch and Toon (1973) to study the evolution of $\mathrm{CO}_{2}$ on Mars from the end of the heavy bombardment period to the present by considering chemical weathering, regolith uptake, polar cap formation, and atmospheric escape by sputtering. They argued that the polar caps could have profound effect on behavior of the system: once ice caps were formed, the volume of ice caps increases drastically owing to a feedback effect between surface pressure, greenhouse effect, and heat transport, resulting in a collapse (catastrophic reduction) of the atmosphere. However, they did not discuss multiplicity of the steady state solutions and stability of them, and, because their model is also zero-dimensional, they could not consider areal extent of polar caps which should affect both the energy balance and the condition for ice cap formation.

In this paper, we investigate stability and property of steady state solutions of the Martian climate system. We consider an atmosphere-ice cap-regolith (AIR) system as a climate system of Mars. According to Hoffert et al. (1981) and Postawko and Kuhn (1986), atmospheric heat transport controls surface temperature profile, hence areal extent of the $\mathrm{CO}_{2}$ caps. Therefore, we construct a one-dimensional energy balance climate model combined with greenhouse effect of $\mathrm{CO}_{2}$ and latitudinal heat transport so that we can express a latitudinal temperature gradient and a change of the areal extent of polar ice caps explicitly. By considering both the energy balance and the $\mathrm{CO}_{2}$ budget, we examine the steady state and stability of the AIR system. Possible scenarios for the evolution of Martian climate system will be also discussed.

\section{Model}

We introduce a one-dimensional energy balance climate model (1-D EBM) based on North et al. (1981). It is, however, noted that the model must be modified in order to describe the climate system of Mars which is quite different from that of the Earth. In this study, we assume annual mean conditions and symmetrical hemispheres in order to compare the previous zero-dimensional annual mean model. The $\mathrm{CO}_{2}$ ice sheet expands from the pole to a certain latitude (this ice cap edge is called "iceline"), and there is no ice from the iceline to Equator.

The 1-D EBM is an energy balance model that can express latitudinal heat transport. In a steady state, the energy balance at the surface of the $i$-th latitude is represented as [Net horizontal heat transport out $]_{i}+[$ Outgoing infrared radiation $_{i}=[\text { Incident solar radiation absorbed }]_{i}$. This may be represented mathematically by the relation

$$
\begin{gathered}
-\frac{\partial}{\partial x}\left[D\left(1-x^{2}\right) \frac{\partial T(x)}{\partial x}\right]+I(x) \\
=\frac{Q}{4} S(x)\left\{1-a\left(x, x_{s}\right)\right\}
\end{gathered}
$$

where $D$ is a thermal diffusion coefficient, $x$ is the sine of latitude (that is, $x=\sin \phi$ where $\phi$ is the latitude), $x_{s}$ cor- responds to the iceline, $T(x)$ is the surface temperature in a given latitude band, $I(x)$ is the outgoing infrared radiation, $Q$ is the solar constant at the orbit of the planet, $S(x)$ is the annual mean solar income distribution, and $a$ is the planetary albedo. In this study, we assume the present solar income distribution as follows (Hoffert et al., 1981).

$$
S(x)=1.235-0.693 x^{2} .
$$

The boundary conditions for symmetric solutions are given by

$$
-D \sqrt{1-x^{2}} \frac{\partial T(x)}{\partial x}=0 \text { for } x=0,1 .
$$

The planetary albedo $a\left(x, x_{s}\right)$ is expressed as follows.

$$
a\left(x, x_{s}\right)= \begin{cases}a_{f} & \text { for } 0<x<x_{s} \\ a_{i} & \text { for } x_{s} \leq x<1\end{cases}
$$

We assumed $a_{f}=0.21$ as the surface albedo of land (Pollack et al., 1987), and $a_{i}=0.65$ as $\mathrm{CO}_{2}$ ice (Leighton and Murray, 1966).

One of the most important problem to study the stability of $\mathrm{CO}_{2}$ atmosphere is condensation of $\mathrm{CO}_{2}$ to form $\mathrm{CO}_{2}$ clouds (Kasting, 1991). Kasting (1991) argued that condensation of $\mathrm{CO}_{2}$ should release latent heat and so decreases the lapse rate, resulting in a decrease in the surface temperature in order to maintain energy balance. The $\mathrm{CO}_{2}$ cloud is a good scatterer for solar radiation, thus it should increase the planetary albedo. Condensation of $\mathrm{CO}_{2}$ may limit the amount of atmospheric $\mathrm{CO}_{2}$ when the entire atmosphere is covered with $\mathrm{CO}_{2}$ cloud.

Recently, Forget and Pierrehumbert (1997) argued that $\mathrm{CO}_{2}$ ice particle larger than $10 \mu \mathrm{m}$ can scatter infrared radiation back to the surface. They included this effect in the 1-D radiative-convective model designed for early Mars by Kasting (1991), and showed that greenhouse effect of $\mathrm{CO}_{2}$ cloud could be quite powerful. Although magnitude of the greenhouse effect of $\mathrm{CO}_{2}$ cloud should depend on fraction of cloud cover and optical thickness, these properties have not been known. Therefore, the effect of $\mathrm{CO}_{2}$ clouds on the energy balance may have been still unclear.

In this study, we adopt a traditional expression of outgoing infrared radiation $I(x)$ which is approximated to a linear function of $T(x)$ as follows:

$$
I(x)=A+B T(x) .
$$

The coefficients $A$ and $B$ depend on the atmospheric $\mathrm{CO}_{2}$ pressure. We determined these coefficients on the basis of the radiative-convective calculations by Pollack et al. (1987) which include greenhouse effects of $\mathrm{CO}_{2}$ and $\mathrm{H}_{2} \mathrm{O}$. Although the model of Pollack et al. (1987) neglects $\mathrm{H}_{2} \mathrm{O}$ clouds, effects of $\mathrm{H}_{2} \mathrm{O}$ clouds should be small when the surface temperature is below $273 \mathrm{~K}$ (the condition considered mainly in this study). Their model also neglects effects of the $\mathrm{CO}_{2}$ clouds. However, we will also discuss the case for $\mathrm{CO}_{2}$ cloud formation (Kasting, 1991; Forget and Pierrehumbert, 1997) in the later section. We parameterize $A$ and $B$ as a function of $\rho\left(=\log P_{\text {air }}: P_{\text {air }}\right.$ is the surface pressure of $\mathrm{CO}_{2}$ (in bar)), and change them at $T_{0}$ for the most appropriate fitting 
as follows:

$$
\begin{gathered}
I=\left\{\begin{array}{l}
A_{1}+B_{1} T \text { for } T>T_{0} \\
A_{2}+B_{2} T \text { for } T \leq T_{0}
\end{array}\right. \\
\left\{\begin{array}{l}
A=a_{1} \rho^{4}+a_{2} \rho^{3}+a_{3} \rho^{2}+a_{4} \rho+a_{5}\left(\mathrm{~W} / \mathrm{m}^{2}\right) \\
B=b_{1} \rho^{4}+b_{2} \rho^{3}+b_{3} \rho^{2}+b_{4} \rho+b_{5}\left(\mathrm{~W} / \mathrm{m}^{2} \mathrm{~K}\right) .
\end{array}\right.
\end{gathered}
$$

Fitting parameters $\left(a_{1} \sim a_{5}, b_{1} \sim b_{5}\right.$, and $\left.T_{0}\right)$ are shown in Table 1.

According to Gierasch and Toon (1973), we assume that heat is transported meridionally by baroclinic instability. We adopt the parameterization of Stone (1972) for vertically integrated heat flux which is expressed as a linear function of surface pressure. We also assume that $D$ is independent of latitude. Then we obtain

$$
D=\alpha P_{\text {air }}
$$

where $\alpha$ is a constant which is determined by the present-day condition.

The atmospheric pressure of $\mathrm{CO}_{2}$ should change owing to changes in the areal extent of ice caps and the amount of $\mathrm{CO}_{2}$ adsorbed in the regolith. The amount of $\mathrm{CO}_{2}$ adsorbed in the regolith $\left(P_{\text {rego }}\right)$ is represented by the equation used in McKay et al. (1991). However, we modify their expression to consider latitudinal dimension as follows:

$$
P_{\text {rego }}=C \int_{0}^{x_{s}} e^{-T(x) / T_{d}} P_{\text {air }}^{\gamma} d x
$$

where $C$ is a constant normalized by depth of the regolith, and $T_{d}$ and $\gamma$ are parameters that determine the response of adsorption to surface temperature and pressure, respectively. The values of these parameters are adopted from McKay et al. (1991).

The amount of $\mathrm{CO}_{2}$ as polar ice caps $\left(P_{\text {ice }}\right)$ is estimated from the areal extent of ice caps and assumed $\mathrm{CO}_{2}$ content in ice caps per unit area. The latitude of iceline is determined from the temperature distribution obtained from the energy balance. The condition for the surface temperature at the iceline is defined here as

$$
T\left(x_{s}\right)=T_{\text {sub }}\left(P_{\text {air }}\right)
$$

where $T_{\text {sub }}$ represents freezing point of $\mathrm{CO}_{2}$ as a function of the atmospheric pressure. Although $\mathrm{CO}_{2}$ ice disappears in summer on the north pole while it remains throughout the year on the south pole on Mars at present (James et al.,

Table 1. Fitting parameters for greenhouse model of Pollack et al. (1987).

\begin{tabular}{cccll}
\hline \multicolumn{5}{c}{$T_{0}=230.1 \mathrm{~K}$} \\
\hline$i$ & $a_{i}\left(A_{1}\right)$ & $a_{i}\left(A_{2}\right)$ & $b_{i}\left(B_{1}\right)$ & $b_{i}\left(B_{2}\right)$ \\
\hline 1 & 0.6449 & 0.1068 & -0.003256 & -0.00094 \\
2 & 13.28 & 2.198 & -0.06758 & -0.0195 \\
3 & 99.54 & 16.48 & -0.5069 & -0.1464 \\
4 & 329.9 & 54.64 & -1.68 & -0.485 \\
5 & -372.7 & -61.72 & 1.898 & 0.5479 \\
\hline
\end{tabular}

1992; Tanaka and Abe, 1991), we assume symmetry of hemisphere for simplicity. It is noted that the correct amount of this reservoir may have been still unclear. According to the previous studies, the amount of $\mathrm{CO}_{2}$ in the ice-cap reservoir is estimated to be from several mbars (Fanale et al., 1982) to $<254$ mbars (more realistically, <a few tens of mbars) (Mellon, 1996). Therefore, the amount of $\mathrm{CO}_{2}$ in the ice-cap reservoir may be less than a few tens of mbars, or, at least, several mbars. In this study, we assume annually averaged expansion of the $\mathrm{CO}_{2}$ caps to be as far as $70^{\circ}$ for both caps with a thickness of $25 \mathrm{~m}$, then we obtain $P_{\text {ice }}=85 \mathrm{mbar}$ for a nominal current condition. The value we used can be regarded as an upper estimate for the $\mathrm{CO}_{2}$ ice-cap reservoir.

When we give the total amount of $\mathrm{CO}_{2}$ contained in the AIR system $\left(P_{\text {total }}\right)$, that is,

$$
P_{\text {total }}=P_{\text {air }}+P_{\text {ice }}+P_{\text {rego }}
$$

we can obtain steady state solutions for the system by solving the energy balance and the $\mathrm{CO}_{2}$ exchange among the reservoirs.

\section{Results}

\subsection{Steady state solutions of the 1-D EBM}

In this section, we show steady state solutions of the 1-D EBM and discuss some specific features. Figure 1 shows steady state solutions of the 1-D EBM (Eqs. (1) (8), and (10)) expressed as a curved surface of

$$
S_{\mathrm{eff}} \equiv \frac{Q}{Q_{0}}=f\left(P_{\mathrm{air}}, \phi_{s}\right)
$$

where $S_{\text {eff }}$ is an effective solar constant and $Q_{0}$ is the solar constant at the Martian orbit today. Steady state solutions of the AIR system (Eqs. (1) (11)) exist on this curved surface. Figure 2(a) is a plane of $\phi_{s}=90^{\circ}\left(P_{\text {air }}-S_{\text {eff }}\right.$ plane $)$ of Fig. 1. The region above the curve is the ice-free solutions, and there is no solution below the curve. Figure 2(b) is a projection

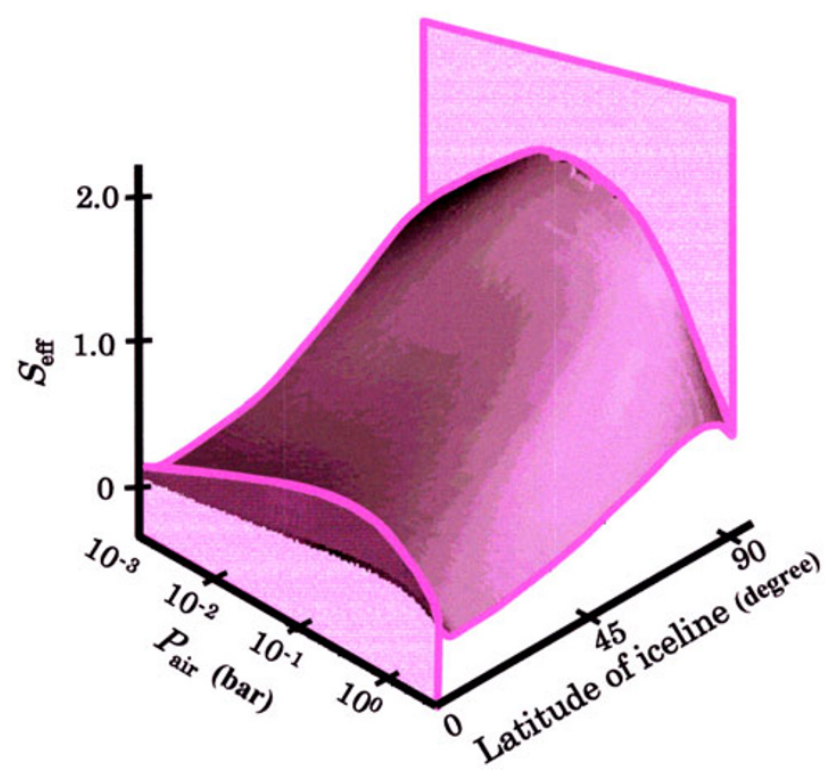

Fig. 1. Steady state solutions of the EBM. 
(a)

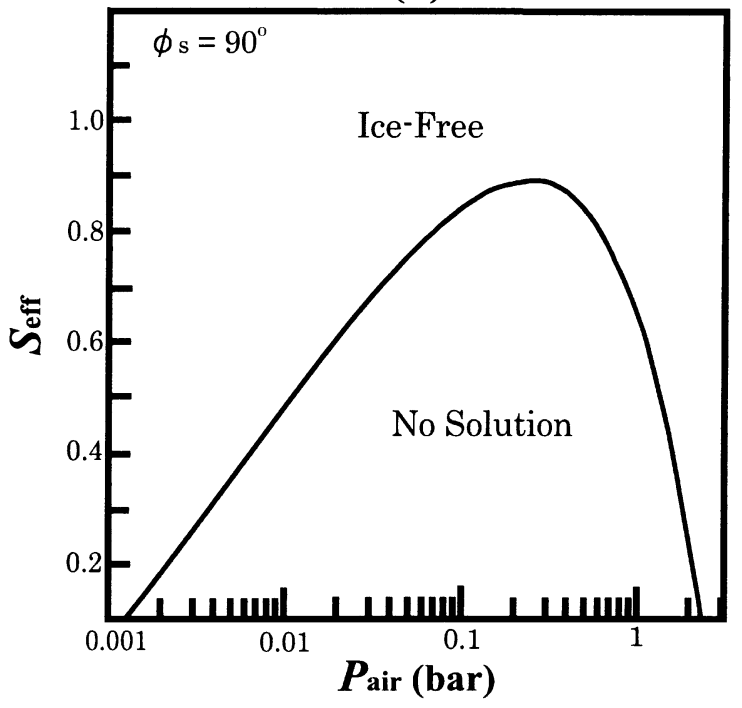

(b)

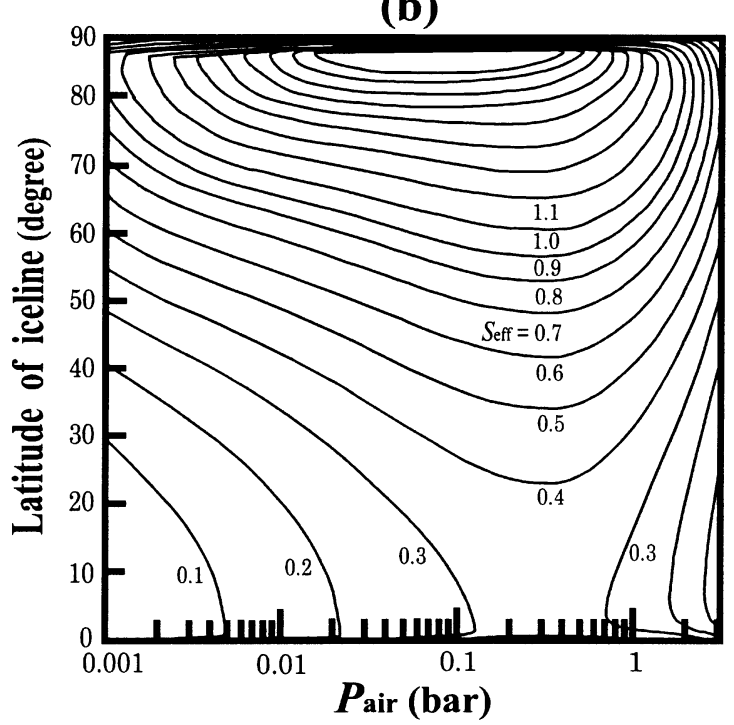

(c)

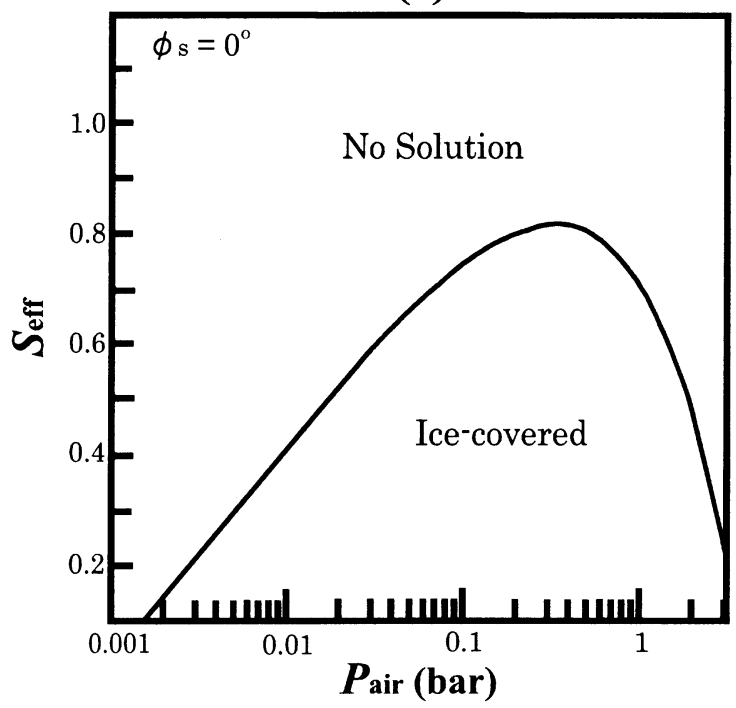

Fig. 2. Cross sections of Fig. 1. (a) A plane of $\phi_{s}=90^{\circ}$. The region above the curve is the ice-free solutions. The sign of $\partial S_{\text {eff }} / \partial P_{\text {air }}$ changes because $P_{\text {air }}$ affects both the greenhouse effect and the freezing point of $\mathrm{CO}_{2}$. (b) The projection of the solutions onto $P_{\text {air }}-\phi_{s}$ plane. (c) A plane of $\phi_{s}=0^{\circ}$. The region below the curve is the ice-covered solutions. of Fig. 1 onto $P_{\text {air }}-\phi_{s}$ plane. Figure 2(c) is a plane of $\phi_{s}=$ $0^{\circ}\left(P_{\text {air }}-S_{\text {eff }}\right.$ plane $)$. The region below the curve is the icecovered solutions, and there is no solution above the curve.

In the climate system of the Earth, there exists ice albedo feedback which is a positive feedback mechanisms derived from albedo difference between land and ice caps (Budyko, 1969). In the Martian climate system, however, additional three feedbacks with respect to $\mathrm{CO}_{2}$ ice caps will exist; (1) increase in the atmospheric $\mathrm{CO}_{2}$ pressure due to decrease in the ice caps and decrease in the amount of $\mathrm{CO}_{2}$ adsorbed in the regolith should result in increase in the greenhouse effect and further decrease in the ice caps (this represents a positive feedback), (2) increase in the atmospheric $\mathrm{CO}_{2}$ pressure due to decrease in the ice caps and in the amount of $\mathrm{CO}_{2}$ adsorbed in the regolith should result in increase in freezing point of $\mathrm{CO}_{2}$, which prevents the ice caps from reducing (this represents a negative feedback). We name (1) and (2) "greenhouse feedback" and "freezing point feedback", respectively. Another feedback mechanism is (3) a "heat transport feedback". Change in the atmospheric $\mathrm{CO}_{2}$ pressure should change efficiency of the latitudinal heat transport. When $P_{\text {air }}$ increases, the efficiency of the latitudinal heat transport increases. Then, if the net budget of latitudinal heat transport is positive at the iceline (that is, higher latitude region), the polar caps should shrink. This is a positive feedback mechanism. On the other hand, if the net budget of latitudinal heat transport is negative at the iceline (that is, lower latitude region), the polar caps should expand. In this case, it represents a negative feedback mechanism. This negative feedback was not taken into account in the previous models of Gierasch and Toon (1973) and Haberle et al. (1994), because the ice cap could not extend to lower latitude region in their models.

It is noted that signs of $\partial S_{\text {eff }} / \partial \phi_{s}, \partial S_{\text {eff }} / \partial P_{\text {air }}$, and $\partial \phi_{s} / \partial P_{\text {air }}$ change along the solution surface (Fig. 1). The sign of $\partial S_{\text {eff }} / \partial \phi_{s}$ changes because of the ice albedo feedback (North et al., 1981). On the other hand, signs of $\partial S_{\text {eff }} / \partial P_{\text {air }}$ and $\partial \phi_{s} / \partial P_{\text {air }}$ are determined by relative strength of two feedback effects: freezing point feedback is stronger than greenhouse feedback where $\partial S_{\text {eff }} / \partial P_{\text {air }}>0$ and $\partial \phi_{s} / \partial P_{\text {air }}<0$. This is because, in that region, the ice cap becomes large owing to the higher freezing point when the atmospheric pressure becomes high. On the other hand, greenhouse feedback is stronger where $\partial S_{\text {eff }} / \partial P_{\text {air }}<0$ and $\partial \phi_{s} / \partial P_{\text {air }}>0$. This is because, in that region, the ice cap becomes small owing to the stronger greenhouse effect when the atmospheric pressure becomes high (see Fig. 2).

\subsection{Solutions under the current condition}

Next, we show the steady state solutions of the 1-D EBM coupled with the AIR system (Eqs. (1) (11)). Martian surface condition at present is given as boundary condition. Standard values of the parameters are shown in Table 2.

Figures 3 and 4 show the numerical results. In Fig. 3, the solutions are projected on to the $S_{\text {eff }}-\phi_{s}$ plane. There exists three steady state solutions under the present solar constant $\left(S_{\text {eff }}=1.0\right)$, and we named them from bottom to top, S1, S2, and S3. Globally-ice-covered solutions cannot exist under the present solar constant, because it does not satisfy the balance of the energy budget (see Fig. 2(c)). The solution S1 represents a partial ice-covered solution (ice caps extend to 
Table 2. Standard values of model parameters.

\begin{tabular}{ccl}
\hline Parameter & Value & \multicolumn{1}{c}{ Reference } \\
\hline$Q^{*}$ & $590 \mathrm{~W} / \mathrm{m}^{2}$ & Hoffert et al. $(1981)$ \\
$\phi_{s}^{*}$ & $70^{\circ}$ & James et al. $(1992)$ \\
$P_{\text {air }}^{*}$ & $6 \mathrm{mbar}$ & Pollack et al. $(1987)$ \\
$P_{\text {ice }}^{*}$ & $85 \mathrm{mbar}$ & This study \\
$P_{\text {rego }}^{*}$ & $40 \mathrm{mbar}$ & Zent and Quinn $(1995)$ \\
$T_{d}$ & $35 \mathrm{~K}$ & McKay et al. $(1991)$ \\
$\gamma$ & 0.275 & McKay et al. $(1991)$ \\
$\alpha$ & $5.3 \times 10^{-3} \mathrm{~m} / \mathrm{K} \cdot \mathrm{s}$ & This study \\
\hline
\end{tabular}

*Present-day values.

some latitude) which corresponds to the present state. Fig. 4 shows the steady state solutions on the $P_{\text {air }}-\phi_{s}$ plane. The contours represent the solution for $S_{\text {eff }}$ to be constant on the curved surface of solutions (Fig. 1). The solid line represents a relation between $P_{\text {air }}$ and $\phi_{s}$ under conditions of $S_{\text {eff }}=1.0$ and $P_{\text {total }}=131 \mathrm{mbar}$, but it does not satisfy the temperature condition for the iceline (Eq. (10)), that is, the solid line satisfies Eqs. (1) (9) and (11). Thus, intersections of these two curves represent solutions of the EBM coupled with the AIR system. There are three steady state solutions for EBM under the conditions at present (see Fig. 3). Among these, we suggest that the solution $\mathrm{S} 1$ is stable and the solution $\mathrm{S} 2$ is unstable. This is because the steady state solutions obtained in this study (Fig. 3) is very similar to those obtained in the study for the Earth. According to the discussion on the stability of the climate system of the Earth, when steady state solutions for EBMs are plotted on the solar constant $(Q)$ - the ice line $\left(\phi_{s}\right)$ diagram (like Fig. 3), condition for the positive slope $\left(d Q / d \phi_{s}>0\right)$ of the solution curve is regarded as stable and condition for the negative slope $\left(d Q / d \phi_{s}<0\right)$ is regarded as unstable (e.g., North et al., 1981). This is usually explained intuitively by the relation between change in the solar constant (solar energy input) and direction of areal change of the polar ice cap. When the solution is on the positive slope, the ice cap decreases (increases) as the solar constant increases (decreases). This is physically reasonable. However, when the solution is on the negative slope, the ice cap increases (decreases) while the solar constant increases (decreases). This is physically unreasonable, and, in such a case, the solution is regarded as unstable (North et al., 1981). By analogy with the case for the Earth, we will be able to discuss the stability for the steady state solutions for the climate system of Mars in the same way. Here, "stability" means that, when a small perturbation is given to the solution, the perturbation diminishes for the case of "stable" solution, but it increases for the case of "unstable" solution. In this respect, the above discussion has been proved mathematically for the climate system of the Earth (North et al., 1981). In fact, we can also prove the stability of the steady state solutions for Mars mathematically.

On the other hand, the solution S3 is a stable ice-free (no ice cap) solution which is warmer condition than at present. This is because, when we give a perturbation of the atmospheric pressure to the solution $\mathrm{S} 3$, a state of the system will

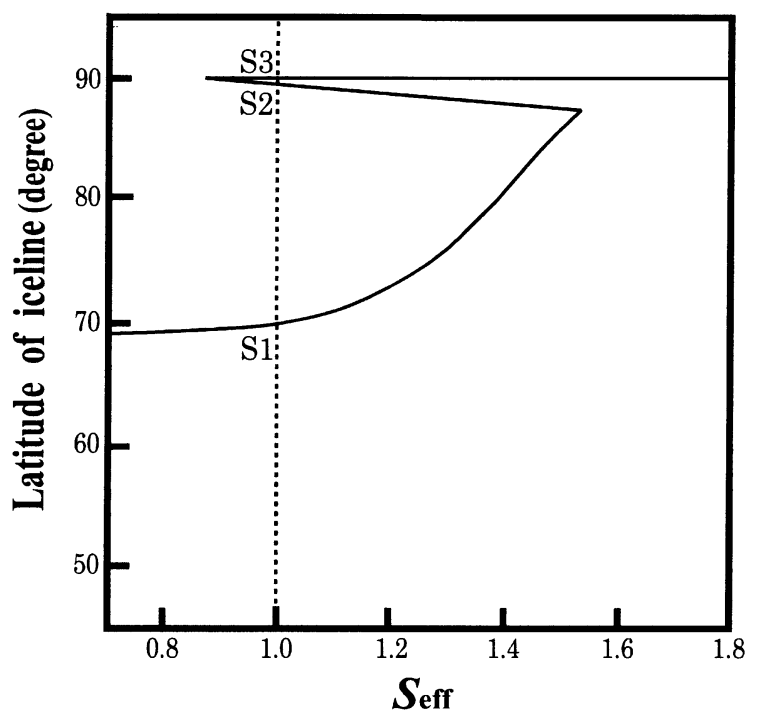

Fig. 3. Steady state solutions of the EBM and the AIR system under the current condition projected onto the $S_{\text {eff }}-\phi_{s}$ plane.

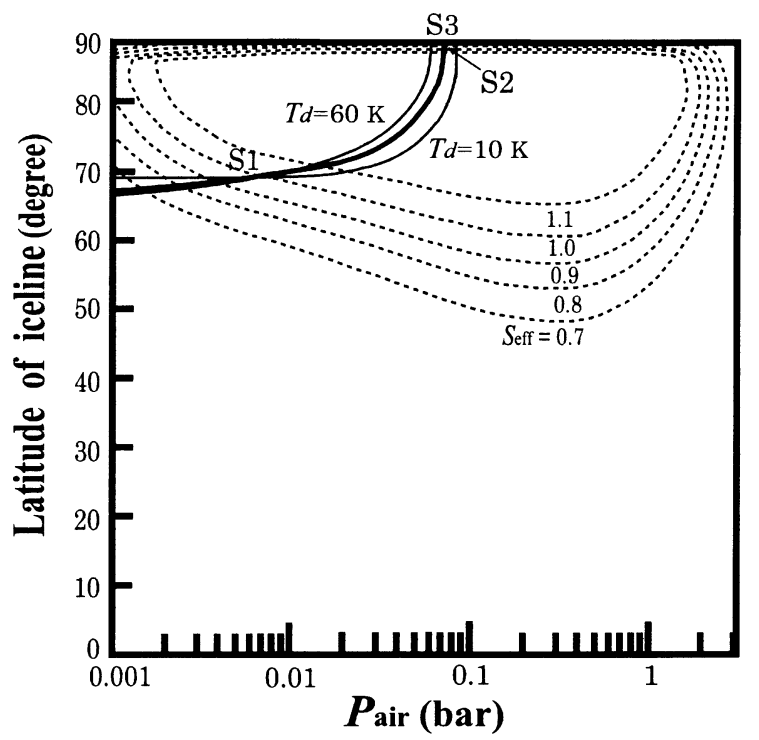

Fig. 4. The projection of the results onto the $P_{\text {air }}-\phi_{s}$ plane. Heavy line represents the result for $S_{\mathrm{eff}}=1.0$ and $P_{\mathrm{total}}=131 \mathrm{mbar}\left(T_{d}=35 \mathrm{~K}\right)$. For comparison, cases for $T_{d}=10 \mathrm{~K}$ and $60 \mathrm{~K}$ are represented as thin lines. Intersections of these curves represent the steady state solutions of the 1-D EBM and the AIR system.

go back to the solution S3 because of a negative feedback mechanism owing to gas exchange between the atmosphere and the regolith which depends on the atmospheric pressure. Therefore, there are two stable solutions for the EBM coupled with the AIR system at the present condition.

Stability of the solutions S1, S2, and S3 can also be shown by numerical simulations. Because characteristic time for a response of the regolith should be much longer than that for a response of $\mathrm{CO}_{2}$ cap stabilization of the atmospheric pressure (Kieffer and Zent, 1992), we deal with the ice caps and the regolith separately. When a small perturbation is given to these steady state solutions, the perturbation decreases with 
time for the case of the solution S1 and S3, but it increases with time for the cases of the solution S2. These results are consistent with the above discussion.

\section{Discussion}

\subsection{Multiple solutions}

The AIR system has two stable steady state solutions (the present state and warmer state) under the present solar constant. This multiplicity seems to be consistent with the results of the previous studies (Gierasch and Toon, 1973; McKay et al., 1991). However, the multiple solutions of this study is derived from the one-dimensional model which considers greenhouse effect of $\mathrm{CO}_{2}$, adsorption of $\mathrm{CO}_{2}$ by the regolith, and the change of the ice cap area. Because Gierasch and Toon (1973) did not consider greenhouse effect of $\mathrm{CO}_{2}$ and the regolith effect, and assumed polar cap as a infinite $\mathrm{CO}_{2}$ reservoir with no areal extent, their solutions are different qualitatively from those obtained in this study. In fact, McKay et al. (1991) showed that the warmer solution of Gierasch and Toon (1973) should disappear when greenhouse effect of $\mathrm{CO}_{2}$ is considered in the model.

On the other hand, McKay et al. (1991) considered greenhouse effect of $\mathrm{CO}_{2}$ and adsorption of $\mathrm{CO}_{2}$ by the regolith, but they did not consider effects of $\mathrm{CO}_{2}$ ice caps. McKay et al. (1991) also obtained multiple solutions by using their model although existence of the warmer solution depends strongly on value of the parameter $T_{d}$. They assumed that the amount of $\mathrm{CO}_{2}$ reserved in the atmosphere and the regolith is constant. However, at the solutions S1 and S3 obtained in this study, the total amount in these two reservoirs is different from each other because of contribution of the ice cap reservoir (although the difference might be small). Therefore, the multiple solutions obtained by McKay et al. (1991) are not exactly the same as S1 and S3. Although the amount of ice cap reservoir might be small, this results in an essential difference in the multiplicity of the solutions. McKay et al. (1991) argued that whether multiple solutions exist or not depends strongly on the parameter $T_{d}$. Results of this study, however, indicate that multiple solutions exist regardless of the $T_{d}$ value for $10 \mathrm{~K} \sim 60 \mathrm{~K}$ (the range McKay et al. (1991) examined), as shown in Fig. 4. This is because difference in the amount of $\mathrm{CO}_{2}$ ice caps results in different $P_{\text {air }}-T$ relation. Thus, we obtain two pairs of lines (one solid line and one dashed line, see Fig. 5), although McKay et al. (1991) considered only one pair of lines. Because each pair has one intersection, there are always two solutions in our model. Our results are also relatively insensitive to the parameter $\gamma$. It should be noted that, McKay et al. (1991) as well as Gierasch and Toon (1973) studied multiple solutions only at the present time, and it is impossible to consider a condition under which there are larger $\mathrm{CO}_{2}$ ice caps which might have existed on the early Mars.

Haberle et al. (1994) also studied the AIR system. They suggested that more than one kind of scenarios can exist on initial state of Mars, although they did not discuss multiple steady state solutions and stability of the solutions. On the other hand, Haberle et al. (1994) argued that a collapse according to a discontinuous jump from a steady state solution to another could occur in the AIR system. In order to deal with this issue in our model, we consider the evolution of the

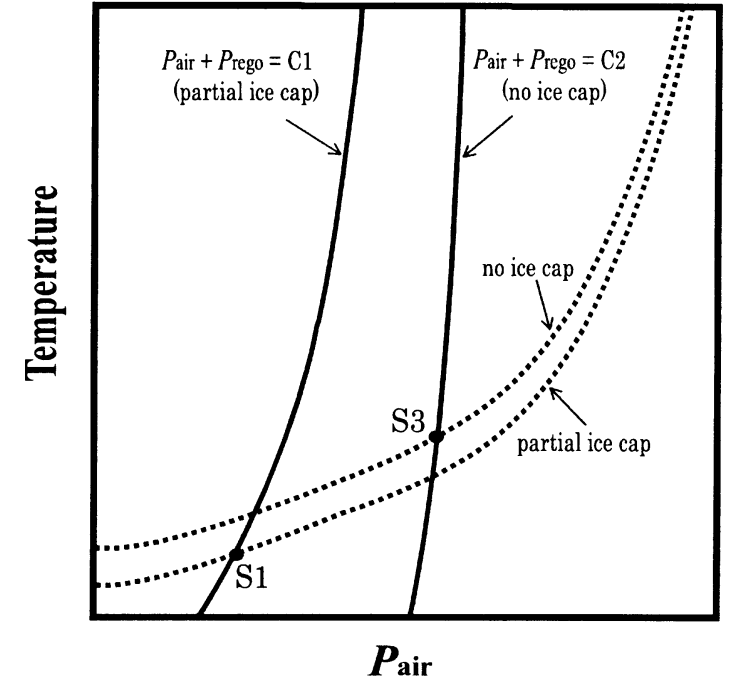

Fig. 5. A schematic illustration of $P-T$ diagram which appeared in McKay et al. (1991). The dashed lines show an average temperature of Mars as a function of $P_{\text {air }}$ with partial ice cap (the present condition) and with no ice cap. The solid lines represent regolith adsorption curves for $P_{\text {air }}+P_{\text {rego }}=C_{1}$ and $C_{2}\left(C_{1}<C_{2}\right)$. Each solid line has one intersection, that is, there are two steady state solutions irrespective of the parameter value.

AIR system.

\subsection{Evolution of Martian climate}

According to stellar evolution models, the solar luminosity has increased since the solar system was formed (e.g., Gough, 1981). Therefore the horizontal axis of Fig. 3 can be regarded as the time axis, and to trace the curve leftward from the present state (S1) should correspond to the Martian climate history tracing back to the past. Because the solar luminosity would have been lower in the past, the surface temperature would have been lower, much more $\mathrm{CO}_{2}$ would have condensed to expand the ice sheet, and the regolith would have adsorbed more $\mathrm{CO}_{2}$. Therefore, in this respect, the atmospheric $\mathrm{CO}_{2}$ pressure should have been lower in the past. On the other hand, the Martian $\mathrm{CO}_{2}$ atmosphere might have decreased during the history by some removal processes (e.g., Melosh and Vickery, 1989; Brain and Jakosky, 1998; Luhmann et al., 1992; Pollack et al., 1987). If the Martian climate was warm and wet in the past because of strong greenhouse effect of $\mathrm{CO}_{2}$, the amount of atmospheric $\mathrm{CO}_{2}$ should have been larger than that at present. Then the implication for the amount of $\mathrm{CO}_{2}$ to have decreased in the past as noted above seems to contradict the past warm climate.

This apparent contradiction results from the assumption for the total amount of $\mathrm{CO}_{2}$ in the AIR system to have been constant. When we consider the evolution of Martian climate system, the total amount of $\mathrm{CO}_{2}$ within the system should change. For example, if there existed liquid water, the atmospheric $\mathrm{CO}_{2}$ would be consumed through silicate weathering followed by precipitation of carbonate minerals (Pollack et al., 1987). It is also suggested that the atmospheric $\mathrm{CO}_{2}$ has escaped owing to impact erosion of the atmosphere by cometary impacts during the heavy bombardment period (Melosh and Vickery, 1989) and sputtering of reentering $\mathrm{O}^{+}$pickup ions over the history of Mars (Luhmann 
et al., 1992).

In order to consider the evolution of Martian climate system, we assume the following two extreme scenarios for the removal of $\mathrm{CO}_{2}$ from the AIR system.

Case I: A large amount of $\mathrm{CO}_{2}$ was removed from the system in a very short period early in the Martian history, and $\mathrm{CO}_{2}$ did not decrease after that event.

Case II: The amount of total $\mathrm{CO}_{2}$ in the system has decreased gradually during the history of Mars after the end of the heavy bombardment period.

In both cases, we assume that the early Martian climate was warm and wet because of greenhouse effect of $\mathrm{CO}_{2}$, and consider how the Martian climate evolves to the present condition. The hystereses of the steady state solution are shown in Fig. 6. For example, when $S_{\text {eff }}=0.7$ and $P_{\text {total }}=4$ bars, there is one stable steady state solution "A". This is the ice-free solution but liquid water can exist only in the equatorial region (the atmospheric $\mathrm{CO}_{2}$ pressure is about 3.5 bars). Then, as some amount of $\mathrm{CO}_{2}$ was removed from the AIR system due to impact erosion and/or weathering followed by carbonate precipitation, the total amount of $\mathrm{CO}_{2}$ would decrease. In this case, the time scale for decrease in $\mathrm{CO}_{2}$ would be much shorter than that for increase in the solar luminosity. The solution A would move along the curve leftward with time, and finally reach another solution $\mathrm{A}^{\prime}$ where $P_{\text {total }}=1.35$ bars. If the total amount of $\mathrm{CO}_{2}$ decreases furthermore, the Martian climate state should jump to the partial ice-covered solution " $B$ ". This climate jump results in a drastic decrease in the atmospheric pressure and an expansion of the ice caps to the mid-latitude. Both the ice albedo and the

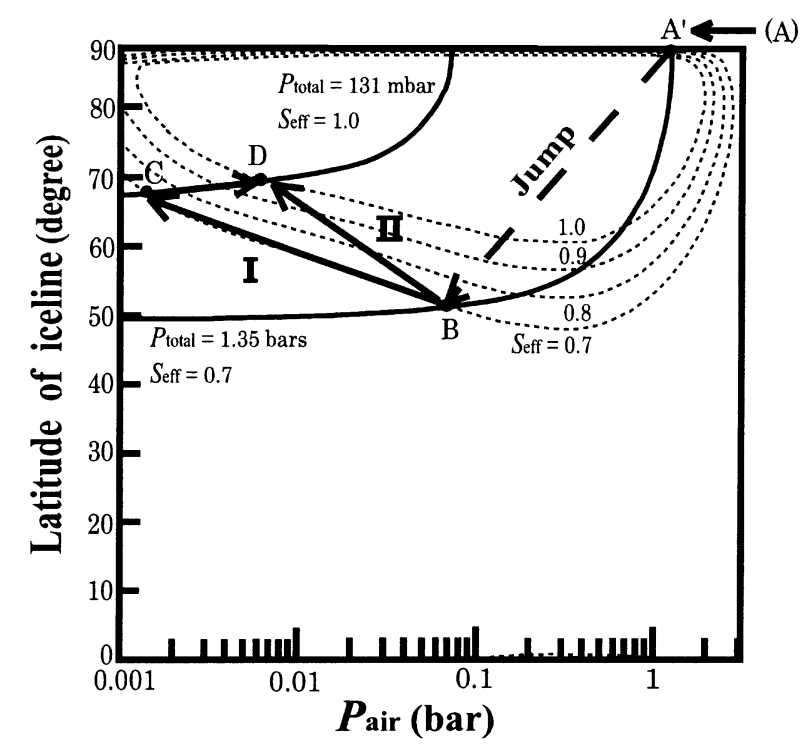

Fig. 6. The climate jump and the following two extreme scenarios for the evolution of the Martian surface environment. Case I: a large amount of $\mathrm{CO}_{2}$ was removed from the system in a very short period early in the Martian history $\left(\mathrm{A} \rightarrow \mathrm{A}^{\prime} \rightarrow \mathrm{B} \rightarrow \mathrm{C}\right.$ ), and then, the total $\mathrm{CO}_{2}$ has been constant $(\mathrm{C} \rightarrow \mathrm{D})$. Case II: after the end of the heavy bombardment period $\left(\mathrm{A} \rightarrow \mathrm{A}^{\prime} \rightarrow \mathrm{B}\right)$, the amount of total $\mathrm{CO}_{2}$ in the system has decreased gradually during the history of Mars $(\mathrm{B} \rightarrow \mathrm{D})$. In both cases, climate jump $\left(\mathrm{A}^{\prime} \rightarrow \mathrm{B}\right)$ should occur in its earliest history. greenhouse feedbacks play significant roles in the climate jump: once ice caps were formed, the atmospheric pressure, so the greenhouse effect and the heat transport should decrease, but ice cap formation should increase the planetary albedo. Therefore, formation of the ice caps resulted in further cooling of the polar region. This climate jump is essentially the same as a "collapse" proposed by Haberle et al. (1994). However, behaviors of the atmospheric $\mathrm{CO}_{2}$ pressure through and after the event are different: at the end of the climate jump (solution B), $P_{\text {air }}$ is higher than that at present in this model, although it is lower than that at present at the end of the collapse in the model of Haberle et al. (1994). This is because the model of Haberle et al. (1994) cannot express a change of the areal extent of polar ice caps although condition for the freezing point is quite different between at the pole and at the actual iceline. In this respect, our solution is different from that of Haberle et al. (1994). The steady state solution B is too cold for liquid water to exist. Thus, effective decrease in the total $\mathrm{CO}_{2}$ by precipitation of carbonate minerals cannot occur hereafter.

In the Case I, the amount of the $\mathrm{CO}_{2}$ atmosphere could have decreased during short period compared with the timescale of solar luminosity change $(\mathrm{B} \rightarrow \mathrm{C})$. Then, the solution may have approached the present state with an increase in the solar luminosity $(\mathrm{C} \rightarrow \mathrm{D})$. It is noted that in Fig. 6 the atmospheric pressure at the point $\mathrm{B}(60 \mathrm{mbars})$ is much higher than that at the point $\mathrm{C}(\sim 1.5 \mathrm{mbars})$. This means not only that the greenhouse effect at the point $\mathrm{B}$ is stronger than that at the point $\mathrm{C}$, but also that the freezing point at the point $\mathrm{B}$ is much higher than that at point $\mathrm{C}$. In this case (evolution from the point $B$ to the point $C$ in Fig. 6), the effect of decrease in the freezing point exceeds the effect of decrease in the greenhouse effect. As a result, considering the pressure-temperature conditions at the ice line, the ice line retreats to the higher latitude while the $\mathrm{CO}_{2}$ level (that is, the global surface temperature) is lower at the point $C$ than at the point B, even when the solar luminosity is constant. This is one of the most interesting results in this study. On the other hand, in the Case II, the amount of $\mathrm{CO}_{2}$ decreased on a timescale comparable to the solar luminosity change $(\mathrm{B} \rightarrow \mathrm{D})$.

As mentioned above, Haberle et al. (1994) did not referred to either the $\mathrm{B} \rightarrow \mathrm{C}$ path or the $\mathrm{B} \rightarrow \mathrm{D}$ path, because $P_{\text {air }}$ must increase after the collapse in a scenario derived from their model. In both cases, if past Martian climate was warm and wet owing to the greenhouse effect of $\mathrm{CO}_{2}$, the climate jump must have occurred during the evolution to the present state, and $\mathrm{CO}_{2}$ ice caps on the early Mars could have extended to the mid-latitude. It is noted that, even if the atmospheric $\mathrm{CO}_{2}$ pressure decreases from the ice-free state, $\mathrm{CO}_{2}$ ice caps cannot be formed directly from that state when $S_{\text {eff }}$ is larger than 0.88 (see Fig. 2(a)). Therefore, if it were the case, the climate jump must have occurred before $1.6 \mathrm{Gyr}\left(S_{\mathrm{eff}}=0.88\right)$ (Gough, 1981). It might be consistent with the suggestion for the existence of the Austral ice sheet which might have extended to the mid-latitude of the southern hemisphere on Mars during the Hesperian age (Baker et al., 1991).

It is noted that there must have been a supply of $\mathrm{CO}_{2}$ via volcanism, so the total amount of $\mathrm{CO}_{2}$ may not have decreased throughout the Martian history. Haberle et al. (1994) 
argued that there is no solution of which the total amount of $\mathrm{CO}_{2}$ have decreased throughout the Martian history to reach the present condition. On the other hand, using the same model with Haberle et al. (1994), Gulick et al. (1997) suggested that pulses of $\mathrm{CO}_{2}$ injected into the atmosphere more recently than 4 Gyr can place the atmosphere into a stable, higher pressure, warmer state. At any rate, accurate evaluation of processes which control the amount of $\mathrm{CO}_{2}$ in the AIR system should be required.

In this study, formation of $\mathrm{H}_{2} \mathrm{O}$ ice caps is not considered. $\mathrm{H}_{2} \mathrm{O}$ ice caps might have affected the evolution of Mars from warm and wet condition to cold condition. However, $\mathrm{H}_{2} \mathrm{O}$ ice caps must have formed before the formation of $\mathrm{CO}_{2}$ ice caps. Thus, behaviors of $\mathrm{CO}_{2}$ ice caps can be discussed, at least qualitatively, even if we do not consider formation of $\mathrm{H}_{2} \mathrm{O}$ ice caps. However, if $\mathrm{H}_{2} \mathrm{O}$ ice caps exists, the temperature in the polar region is lower than that with no $\mathrm{H}_{2} \mathrm{O}$ ice caps owing to its high albedo. In such a case, $\mathrm{CO}_{2}$ ice caps are formed under the higher solar constant than that predicted in this study.

\subsection{Problem on $\mathrm{CO}_{2}$ clouds}

According to Kasting (1991), when $\mathrm{CO}_{2}$ condensation is included in the radiative-convective equilibrium model, the result differs remarkably from that of Pollack et al. (1987), especially for $S_{\text {eff }}=0.7$ or 0.8 . The greenhouse effect should be reduced when $P_{\text {air }}$ is above a few hundreds mbar because of formation of $\mathrm{CO}_{2}$ clouds, and what is worse, $\mathrm{CO}_{2}$ condensation limits the amount of atmospheric $\mathrm{CO}_{2}$. On the other hand, Forget and Pierrehumbert (1997) showed that $\mathrm{CO}_{2}$ ice particle larger than $10 \mu \mathrm{m}$ can scatter infrared radiation back to the surface, thus $\mathrm{CO}_{2}$ clouds may have powerful greenhouse effect. Because the effect of $\mathrm{CO}_{2}$ clouds on the energy balance may have been still unclear, the results shown here might be tentative. Therefore, we also consider the cases in which $\mathrm{CO}_{2}$ cloud formation is included in the model (Fig. 7).

Figure 7(a) shows the solutions for the case which considers effects of $\mathrm{CO}_{2}$ cloud formation on the energy balance based on the results of Kasting (1991). In this case, an ice-free solution is impossible during the earliest history of Mars. For an initial state, a partial ice-covered solution (when $P_{\text {total }} \leq 3$ bars) or ice-covered solution (when $P_{\text {total }}>3$ bars) is expected. If the initial state was ice-covered, ice at the equatorial region should have disappeared during the evolution as $S_{\text {eff }}$ increased, and the steady state should have jumped to a partial ice-covered state to reach the present condition.

Figure 7(b) shows the solutions for the case based on the results of Forget and Pierrehumbert (1997) with radii of the $\mathrm{CO}_{2}$ ice particles are $50 \mu \mathrm{m}$, the optical depth is 10 , and dry atmosphere (note that they provided the results only above $P_{\text {air }}=100$ mbar). As shown in this figure, area of the ice caps at a certain $P_{\text {air }}$ is smaller due to powerful greenhouse effect of $\mathrm{CO}_{2}$ clouds. In this case, if the initial state was ice-free, the solution cannot jump until $P_{\text {air }}$ decrease to, at least, about 100 mbar, although we cannot know where the jump will occur because of absence of original data below 100 mbar.

It is expected that $\mathrm{CO}_{2}$ cloud formation has been inhibited in regions of atmospheric subsidence (Forget and Pierrehumbert, 1997). Magnitude of the effect of $\mathrm{CO}_{2}$ clouds should depend on fraction of cloud cover and optical thickness of
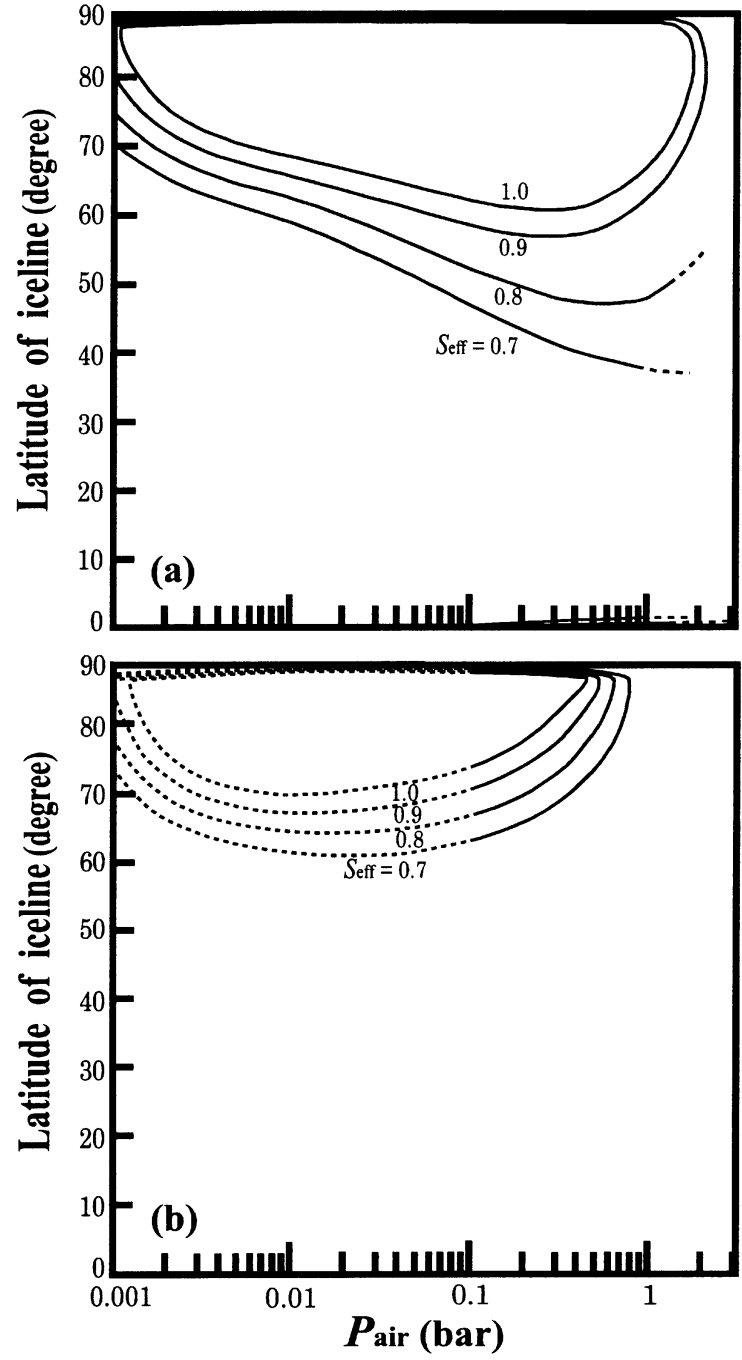

Fig. 7. Steady state solutions of the EBM based on the results of (a) Kasting (1991), and (b) Forget and Pierrehumbert (1997). Dashed curves represent the region where original data is not available.

the $\mathrm{CO}_{2}$ cloud, but these properties have not been known. In order to solve this problem, it is necessary to introduce a full 3-D climate model, but it is beyond the purpose of this study.

\section{Summary}

In this paper, we have discussed the Martian surface environment. We assumed that $\mathrm{CO}_{2}$ has been the dominant constituent of the Martian atmosphere and that the atmosphere, the polar ice caps, and the regolith are major reservoirs of $\mathrm{CO}_{2}$ (the AIR system). In order to study stability and evolution of the AIR system, we introduced a one-dimensional energy balance climate model (EBM) which considers change of the ice cap area, latitudinal heat transport, and greenhouse effect of $\mathrm{CO}_{2}$.

We examined stability of the steady state solutions of the EBM and the AIR system. The stability is determined from competition among four feedback effects (the ice albedo feedback, the greenhouse feedback, the freezing point feedback, and the heat transport feedback). The ice albedo, the greenhouse, and the heat transport feedbacks are positive feedbacks which make the system unstable, but the freezing 
point feedback is negative feedback which makes it stable. When we adopt the present state as a boundary condition and assume the total amount of $\mathrm{CO}_{2}$ in the AIR system to be constant, there exists two stable steady state solutions under the present solar luminosity. One corresponds to a partial icecovered solution (the present state), and the other is a warmer ice-free solution. Although this seems to be consistent with the former results by Gierasch and Toon (1973) and McKay et al. (1991), our result is different qualitatively from their results.

If the Martian climate was warm and wet owing to the greenhouse effect of $\mathrm{CO}_{2}$ in the past, the total amount of $\mathrm{CO}_{2}$ contained in the system must have been much more than that at present. This means that the total amount of $\mathrm{CO}_{2}$ in the system has been decreased by some removal processes. In this case, we found that the climate jump which is a drastic expansion of ice caps and decrease of the atmosphere must have occurred during the evolutionary path from the warm and wet condition to the present state, and ice caps on the early Mars could have extended to the mid-latitude. The atmospheric pressure may have decreased further after the climate jump.

Acknowledgments. The authors are most grateful to Y. Abe and T. Ikeda for helpful discussions and comments on this work. We also thank V. Gulick and an anonymous referee for the helpful reviews.

\section{References}

Baker, V. R., R. G. Storm, V. C. Gulick, J. S. Kargel, G. Komatsu, and V. $\mathrm{S}$. Kale, Ancient oceans, ice sheets and the hydrological cycle on Mars, Nature, 352, 589-594, 1991.

Baker, V. R., M. H. Carr, V. C. Gulick, C. R. Williams, and M. S. Marley, Channels and valley networks, in Mars, edited by H. H. Kieffer et al., pp. 493-522, Univ. of Ariz. Press, Tuscan, 1992.

Brain, D. A. and B. M. Jakosky, Atmospheric loss since the onset of the Martian geologic record: Combined role of impact erosion and sputtering, J. Geophys. Res., 103, 22,689-22,694, 1998.

Budyko, M. I., The effect of solar radiation variations on the climate of the earth, Tellus, 21, 611-619, 1969.

Fanale, F. P. and W. A. Cannon, Exchange of adsorbed $\mathrm{H}_{2} \mathrm{O}$ and $\mathrm{CO}_{2}$ between the regolith and atmosphere of Mars caused by changes in surface insolation, J. Geophys. Res., 24, 3397-3402, 1974.

Fanale, F. P., J. R. Salvail, W. B. Banerdt, and R. S. Saunders, Mars: the regolith-atmosphere-cap system and climate change, Icarus, 50, 381407, 1982.

Forget, F. and R. T. Pierrehumbert, Warming early Mars with carbon dioxide clouds that scatter infrared radiation, Science, 278, 1273-1276, 1997.

Gierasch, P. J. and O. B. Toon, Atmospheric pressure variation and the climate of Mars, J. Geophys. Res., 30, 1502-1508, 1973.

Goldspiel, J. M. and S. W. Squyres, Ancient aqueous sedimentation on Mars, Icarus, 89, 392-410, 1991.

Gough, D. O., Solar interior structure and luminosity variations, Solar Phys., 74, 21-34, 1981

Gulick, V. C., D. Tyler, C. P. McKay, and R. M. Haberle, Episodic oceaninduced $\mathrm{CO}_{2}$ greenhouse on Mars: implications for fluvial valley formation, Icarus, 130, 68-86, 1997.
Haberle, R. M., Early Mars climate models, J. Geophys. Res., 103, 28,46728,479, 1998.

Haberle, R. M., D. Tyler, C. P. McKay, and W. L. Davis, A model for the evolution of $\mathrm{CO}_{2}$ on Mars, Icarus, 109, 102-120, 1994.

Head, J. W., III, H. Hiesinger, M. A. Ivanov, M. A. Kreslavsky, S. Pratt, and B. J. Thomson, Possible ancient oceans on Mars: evidence from Mars Orbiter Laser Altimeter data, Science, 286, 2134-2137, 1999.

Hoffert, M. I., A. J. Callegari, C. T. Hsieh, and W. Ziegler, Liquid water on Mars: an energy balance climate model for $\mathrm{CO}_{2} / \mathrm{H}_{2} \mathrm{O}$ atmosphere, Icarus, 47, 112-129, 1981.

James, P. B., H. H. Kieffer, and D. A. Paige, The seasonal cycle of carbon dioxide on Mars, in Mars, edited by H. H. Kieffer et al., pp. 934-968, Univ. of Ariz. Press, Tuscan, 1992.

Kasting, J. F., $\mathrm{CO}_{2}$ condensation and the climate of early Mars, Icarus, 94, $1-13,1991$.

Kasting, J. F., Warming early Earth and Mars, Science, 276, 1213-1215, 1997.

Kieffer, H. H. and A. P. Zent, Quasi-periodic climate change on Mars, in Mars, edited by H. H. Kieffer et al., pp. 1180-1218, Univ. of Ariz. Press, Tuscan, 1992.

Kuhn, W. R. and S. K. Atreya, Ammonia photolysis and the greenhouse effect in the primordial of the Earth, Icarus, 37, 207-213, 1979.

Leighton, R. B. and B. C. Murray, Behavior of carbon dioxide and other volatiles on Mars, Science, 153, 136-144, 1966.

Luhmann, J. G., R. E. Johnson, and M. H. G. Zhang, Evolutionary impact of sputtering of the Martian atmosphere by $\mathrm{O}^{+}$pickup ions, Geophys. Res. Lett., 19, 2151-2154, 1992.

McKay, C. P., O. B. Toon, and J. F. Kasting, Making Mars habitable, Nature, 352, 489-496, 1991.

Mellon, M. T., Limits on the $\mathrm{CO}_{2}$ content of the Martian polar deposits, Icarus, 124, 268-279, 1996.

Melosh, H. J. and A. M. Vickery, Impact erosion of the primordial Martian atmosphere, Nature, 338, 487-489, 1989.

North, G. R., R. F. Cahalan, and J. A. Coakley, Jr., Energy balance climate models, Reviews of Geophysics and Space Physics, 19, 91-121, 1981.

Pollack, J. B., J. F. Kasting, S. M. Richardson, and K. Poliakoff, The case for a wet, warm climate on early Mars, Icarus, 71, 203-224, 1987.

Postawko, S. E. and W. R. Kuhn, Effect of the greenhouse gases $\left(\mathrm{CO}_{2}\right.$, $\mathrm{H}_{2} \mathrm{O}, \mathrm{SO}_{2}$ ) on Martian paleoclimate, Proc. Lunar Planet. Sci. Conf. 16th, Part 2, J. Geophys. Res., 91, suppl., D431-D438, 1986.

Sagan, C. and C. Chyba, The early faint Sun paradox: Organic shielding of ultraviolet-labile greenhouse gases, Science, 276, 1217-1221, 1997.

Sagan, C. and G. Mullen, Earth and Mars: Evolution of atmospheres and surface temperatures, Science, 177, 52-56, 1972.

Smith, D. E., M. T. Zuber, H. V. Frey, J. B. Garvin, J. W. Head, D. O. Muhleman, G. H. Pettengill, R. J. Phillips, S. C. Solomon, H. J. Zwally, W. B. Banerdt, and T. C. Duxbury, Topography of the northern hemisphere of Mars from the Mars Orbiter Laser Altimeter, Science, 279, 1686-1692, 1998

Stone, P. H., A simplified radiative-dynamical model for the static stability of rotating atmospheres, J. Atmos. Sci., 29, 405-418, 1972.

Tanaka, H. M. and Y. Abe, A numerical study of the difference between the south and north $\mathrm{CO}_{2}$ polar caps on Mars, Proc. Lunar Planet. Sci., XXIV, 1-7, 1991

Toon, O. B., J. B. Pollack, W. Ward, J. A. Burns, and K. Bilski, The astronomical theory of climate change on Mars, Icarus, 44, 552-607, 1980.

Zent, A. P. and R. C. Quinn, Simultaneous adsorption of $\mathrm{CO}_{2}$ and $\mathrm{H}_{2} \mathrm{O}$ under Mars-like conditions and application to the evolution of the Martian climate, J. Geophys. Res., 100, 5341-5349, 1995.

T. Nakamura (e-mail: kijun@sys.eps.s.u-tokyo.ac.jp) and E. Tajika (e-mail: tajika@eps.s.u-tokyo.ac.jp) 